\title{
Chance-constrained Optimization for Pension Fund Portfolios in the Presence of Default Risk
}

\author{
Yufei Sun ${ }^{\mathrm{a}}$, Grace $\mathrm{Aw}^{\mathrm{a}}$, Ryan Loxton ${ }^{\mathrm{a}, \mathrm{b}, *}$, Kok Lay Teo ${ }^{\mathrm{a}}$ \\ ${ }^{a}$ Department of Mathematics and Statistics, Curtin University, Australia \\ ${ }^{b}$ Institute of Cyber-Systems and Control, Zhejiang University, China
}

\begin{abstract}
In this paper, we consider the portfolio optimization problem for a pension fund consisting of various government and corporate bonds. The aim of the problem is to maximize the fund's cash position at the end of the time horizon, while allowing for the possibility of bond defaults. We model this problem as a stochastic discrete-time optimal control problem with a chance constraint that ensures all future outgoing commitments can be met with sufficiently high probability. We then introduce a corresponding deterministic formulation that is a conservative approximation of the original stochastic optimal control problem. This approximate problem can be solved using gradient-based optimization techniques. We conclude the paper with a simulation study.
\end{abstract}

Keywords:

Pension fund, portfolio optimization, optimal control, gradient-based optimization

\section{Introduction}

The aim of portfolio optimization is to divide an investor's capital judiciously among various financial securities (stocks, bonds, derivatives, etc.) to achieve an optimal balance between risk and return. First introduced by Markowitz in the early 1950s, portfolio optimization is now a mature research field with considerable practical impact.

Markowitz's original mean-variance model (Markowitz (1959)) is still used as the basis for many new portfolio optimization techniques (see, for example, Smith (1967); Fama (1970); Pliska (1997); Çlikyurt and Öekici (2007)). In this model, the return of each security is viewed as a random variable characterized by two fundamental quantities: the mean (which measures the expected return of the security) and the variance (which measures the risk associated with the security). The goal is to maximize the total expected return subject to a given risk level, or alternatively, minimize the total risk subject to a target rate of return. This risk-return tradeoff is the common theme underpinning all portfolio optimization problems.

\footnotetext{
*Corresponding author: Ryan Loxton, Phone: +61-08-92669218, Fax: +61-08-92663197

Email address: rcloxton@gmail. com (Ryan Loxton)
}

\subsection{Literature review}

The literature on portfolio optimization is dominated by extensions to Markowitz's original one-period model. Early papers discuss the problem of maximizing the expected utility of the investor over a single- or multiperiod investment horizon, with various forms for the utility function (Mossin (1968); Merton (1969)). Recent papers consider more complex problem settings, but still rely on the assumption that there are no outflows from the investment portfolio (Zhang et al. (2009); Van Weert et al. (2010); Çanakoğlu and Öekici (2012)). This assumption is not suitable for retirees and other income investors, who withdraw (rather than reinvest) the returns from their portfolios.

In addition to portfolio outflows, the possibility of asset defaults must also be considered when designing an optimal investment portfolio. Modeling the default risk on debt securities, as well as the pricing and management of such securities, is discussed in various papers (Jarrow and Turnbull (1995); Duffie and Singleton (2003); Lando (2004)). The importance of considering the credit risk of debt securities was highlighted by the 2007-2008 global financial crisis.

Investment portfolios are designed to satisfy various constraints, which are typically defined by the investment strategy. One of the most common constraints is an upper bound on the variance (Schweizer (1995)). 
More recently, the problem of controlling bankruptcy risk in portfolio optimization has attracted considerable attention in the literature (Zhu et al. (2004); Chen and Song (2012)). For example, Zhu et al. (2004) propose a model through which an optimal investment policy can be devised to achieve not only an optimal mean-variance tradeoff, but also a low risk of portfolio bankruptcy. In a related study, Chen and Song (2012) model investment returns using a Markov stochastic framework, and show how to control the bankruptcy risk by restricting the portfolio loss to be less than or equal to a specified percentage of the total wealth at the start of each time period.

\subsection{Contribution of this paper}

The purpose of this paper is to introduce a new model for portfolio optimization based on stochastic discretetime optimal control. Instead of maximizing the terminal wealth of an individual investor, we focus on maximizing the terminal cash balance of a conservative pension fund consisting entirely of government and corporate bonds. The pension fund experiences regular stochastic outflows as retirees leave the workforce and draw on their pensions. Thus, to ensure that the fund can meet all obligations with sufficiently high probability, we impose an all-time chance constraint that forces the fund's cash balance to be above a minimum threshold at all times. In this respect, our paper follows a different approach to others in the literature, by imposing a chance constraint, rather than a bound on the variance.

The bonds in the portfolio are assumed to be held for the entire time horizon. Thus, all investment decision$\mathrm{s}$ are made at the start of the time horizon and active management of the fund is not required. A key feature of our model is that it explicitly considers the risk of bond defaults, which facilitates comparisons between the default risk and no-default risk optimal strategies. Pension funds are designed to operate over a long time horizon, and thus it is essential to consider the risk of bond defaults - indeed, a bond issuer who is reputable now may no longer be reputable in 10 years time.

This paper is organized as follows. In Section 2, we model the portfolio optimization problem by a stochastic discrete-time optimal control problem. In Section 3, we convert this stochastic problem into an approximate deterministic problem, whose feasible region is contained within the feasible region of the original stochastic problem. In Section 4, we develop a gradient-based optimization algorithm for solving the deterministic optimal control problem introduced in Section 3. Section 5 includes simulation results showing that our optimized portfolio outperforms the naive $1 / N$ portfolio strategy, which is considered to be the benchmark standard in the finance literature (Demiguel et al. (2009a,b)). Finally, we conclude the paper in Section 6.

\section{Problem statement}

We consider the portfolio optimization problem for a pension fund consisting of $N$ bonds and operating over a time horizon of $T$ periods. Let $x(t)$ denote the fund's cash level at the end of period $t$. Furthermore, let $\alpha_{i}(t)$ denote the promised payment of a type- $i$ bond at the end of period $t$, and let $\psi(t)$ denote the total pension payments to be made during period $t$. We assume that $\psi(t)$ is a random variable of unknown distribution, but with known mean and covariance information. The mean of $\psi(t)$ is denoted by $\mu(t)$ and the covariance of $\psi\left(t^{\prime}\right)$ and $\psi\left(t^{\prime \prime}\right)$ is denoted by $\sigma\left(t^{\prime}, t^{\prime \prime}\right)$.

The bond allocation in the pension fund must be designed to mitigate the risk of bond defaults. If a bond default occurs, then the pension fund will lose all future coupons as well as the redemption value of the bond. Let $p_{i}(t)$ be the probability that type- $i$ bonds default during period $t$. Furthermore, let $y_{i}(t)$ be a binary state variable, where $y_{i}(t)=1$ if type- $i$ bonds do not default before the end of period $t$, and $y_{i}(t)=0$ otherwise. Then $y_{i}(t)$ can be described by the following discretetime difference equation:

$$
y_{i}(t+1)=S_{i}(t+1) y_{i}(t), \quad t=0, \ldots, T-1,
$$

where $S_{i}(t)$ follows a Bernoulli distribution with parameter $1-p_{i}(t)$, and

$$
y_{i}(0)=1 \text {. }
$$

We assume throughout this section that for any choice of $t^{\prime}$ and $t^{\prime \prime}, S_{i}\left(t^{\prime}\right)$ and $\psi\left(t^{\prime \prime}\right)$ are independent (i.e., the occurrence of bond defaults is independent of the amount of outgoing pensions). Note, however, that the Bernoulli random variables $S_{i}(t)$ and $S_{j}(t)$, which govern whether type- $i$ and type- $j$ bonds default during period $t$, are not necessarily independent: the bonds could default for the same reason (e.g., a common bond issuer going bankrupt). Define

$$
\pi_{i j}(t)=\operatorname{Cov}\left\{S_{i}(t), S_{j}(t)\right\}, \quad i, j=1, \ldots, N .
$$

We assume that these covariances are known.

The fund's cash level is governed by the following 
difference equation:

$$
\begin{array}{r}
x(t+1)=x(t)+\sum_{i=1}^{N} \frac{K u_{i}}{\gamma_{i}} \alpha_{i}(t+1) y_{i}(t+1)-\psi(t+1), \\
t=0, \ldots, T-1
\end{array}
$$

where $N$ is the number of bond types, $K$ is the initial capital available for investment, $\gamma_{i}$ is the price of a type$i$ bond at the beginning of the time horizon, and $u_{i}$ is the fraction of initial capital allocated to type- $i$ bonds.

The allocation fractions $u_{i}, i=1, \ldots, N$, are decision variables to be selected optimally. To ensure sufficient diversification of the portfolio, we impose the following box constraints:

$$
0 \leq u_{i} \leq u_{i}^{\max }, \quad i=1, \ldots, N,
$$

where $u_{i}^{\max }$ is the maximum fraction of initial capital that can be invested in type- $i$ bonds.

Furthermore, since the pension fund cannot invest more than the initial capital $K$,

$$
\sum_{i=1}^{N} u_{i} \leq 1
$$

Let $U$ denote the set of all vectors $u=\left[u_{1}, \ldots, u_{N}\right]^{\top} \in$ $\mathbb{R}^{N}$ satisfying (5) and (6). Any vector $u \in U$ is called an admissible allocation vector.

The initial cash level in the pension fund is

$$
x(0)=K\left[1-\sum_{i=1}^{N} u_{i}\right] .
$$

Constraint (6) ensures that $x(0)$ is always non-negative. Substituting (1) into (4) yields

$$
\begin{aligned}
x(t+1)= & x(t)+\sum_{i=1}^{N} \frac{K u_{i}}{\gamma_{i}} \alpha_{i}(t+1) S_{i}(t+1) y_{i}(t) \\
& -\psi(t+1), \quad t=0, \ldots, T-1 .
\end{aligned}
$$

Equations (1), (2), (7), and (8) constitute a system of discrete-time difference equations.

The pension fund's finances must be sufficiently robust to cope with unexpected increases in outgoing commitments (e.g., if more retirees than normal leave the workforce during a given period). Thus, we require that the fund's cash level be greater than or equal to a pre-set minimum level at all times. This condition can be modelled by the following chance constraint:

$$
\operatorname{Pr}\left\{x(t) \geq x_{\min }\right\} \geq q, t=0, \ldots, T,
$$

where $x_{\min }$ is the pre-set minimum cash balance and $q$ is a given probability level.

The objective is to choose a bond allocation that maximizes the total expected wealth at the end of the planning horizon. Here, total wealth is defined as the fund's final cash level plus the redemption values of all bonds in the fund (except for those bonds that have defaulted). Thus, the objective function is

$$
\begin{aligned}
g(u) & =\mathscr{E}\left\{x(T)+\sum_{i=1}^{N} \frac{K u_{i}}{\gamma_{i}} c_{i} y_{i}(T)\right\} \\
& =\mathscr{E}\{x(T)\}+\sum_{i=1}^{N} \frac{K u_{i}}{\gamma_{i}} c_{i} \mathscr{E}\left\{y_{i}(T)\right\}
\end{aligned}
$$

where $c_{i}$ is the redemption price of a type- $i$ bond and $\mathscr{E}$ denotes the mathematical expectation. Here, we assume that all bonds in the portfolio are redeemed at the end of the planning horizon.

The portfolio optimization problem, which takes default risk into account, may now be stated formally as follows.

Problem P. Given the discrete-time stochastic system (1) and (8) with initial conditions (2) and (7), find an admissible allocation vector $u \in U$ such that the objective function (10) is maximized subject to constraint (9).

Problem $\mathrm{P}$ is a discrete-time stochastic optimal control problem subject to the chance constraint (9). The solution of this problem gives the optimal allocation of each bond type in the pension fund. Because of its stochastic nature, Problem $\mathrm{P}$ cannot be solved using conventional optimal control techniques. Thus, in the next section, we will convert Problem $\mathrm{P}$ into a deterministic problem.

\section{Deterministic approximation}

The dynamic equations in Problem $\mathrm{P}$ involve two types of random variables: $S_{i}(t)$, Bernoulli random variables, and $\psi(t)$, random variables of unknown distribution. Thus, it is impossible to derive an explicit form for the probability distribution of $x(t)$ in Problem $\mathrm{P}$, which makes the chance constraint (9) extremely challenging. We will therefore introduce an approximation for this constraint. We begin with the following result.

Lemma 3.1. For each $i=1, \ldots, N$ and $t=$ $1, \ldots, T, y_{i}(t)$ is a Bernoulli random variable with parameter $\prod_{k=1}^{t}\left(1-p_{i}(k)\right)$.

Proof. The result can be proved easily by induction on $t$. 
From Lemma 3.1, we obtain

$$
\begin{aligned}
\mathscr{E}\left\{y_{i}(t)\right\} & =\prod_{k=1}^{t}\left(1-p_{i}(k)\right) \\
\operatorname{Var}\left\{y_{i}(t)\right\} & =\prod_{k=1}^{t}\left(1-p_{i}(k)\right)\left[1-\prod_{k=1}^{t}\left(1-p_{i}(k)\right)\right]
\end{aligned}
$$

Now, define a new set of parameters $o_{i j}(t), i=$ $1, \ldots, N, j=1, \ldots, N$, as follows:

$$
o_{i j}(t)=\operatorname{Cov}\left\{y_{i}(t), y_{j}(t)\right\}, \quad t=0, \ldots, T .
$$

The values of $o_{i j}(t)$ can be computed according to the following result.

Lemma 3.2. For each $i=1, \ldots, N$, and $j=$ $1, \ldots, N, o_{i j}(t)$ satisfies the discrete-time system

$$
\begin{aligned}
& o_{i j}(t+1) \\
& =\left[\pi_{i j}(t+1)+\left(1-p_{i}(t+1)\right)\left(1-p_{j}(t+1)\right)\right] o_{i j}(t) \\
& +\pi_{i j}(t+1) \prod_{k=1}^{t}\left(1-p_{i}(k)\right) \prod_{k=1}^{t}\left(1-p_{j}(k)\right), \\
& \quad t=0, \ldots, T-1,
\end{aligned}
$$

with initial condition

$$
o_{i j}(0)=0,
$$

where $\pi_{i j}(t)$ is as defined in (3).

Proof. Using (1), we have

$$
\begin{aligned}
o_{i j}(t+1)= & \operatorname{Cov}\left\{y_{i}(t+1), y_{j}(t+1)\right\} \\
= & \mathscr{E}\left\{S_{i}(t+1) y_{i}(t) S_{j}(t+1) y_{j}(t)\right\} \\
& -\mathscr{E}\left\{S_{i}(t+1) y_{i}(t)\right\} \mathscr{E}\left\{S_{j+1}(t) y_{j}(t)\right\} .
\end{aligned}
$$

Since $y_{i}(t)$ and $y_{j}(t)$ are independent of $S_{i}(t+1)$ and $S_{j}(t+1)$, it follows that

$$
\begin{aligned}
o_{i j}( & (t+1) \\
= & \mathscr{E}\left\{S_{i}(t+1) S_{j}(t+1)\right\} \mathscr{E}\left\{y_{i}(t) y_{j}(t)\right\} \\
& -\mathscr{E}\left\{S_{i}(t+1)\right\} \mathscr{E}\left\{S_{j}(t+1)\right\} \mathscr{E}\left\{y_{i}(t)\right\} \mathscr{E}\left\{y_{j}(t)\right\} \\
= & \operatorname{Cov}\left\{S_{i}(t+1), S_{j}(t+1)\right\} \\
& \left.+\mathscr{E}\left\{S_{i}(t+1)\right\} \mathscr{E}\left\{S_{j}(t+1)\right\}\right] \\
& \cdot\left[\operatorname{Cov}\left\{y_{i}(t), y_{j}(t)\right\}+\mathscr{E}\left\{y_{i}(t)\right\} \mathscr{E}\left\{y_{j}(t)\right\}\right] \\
- & \mathscr{E}\left\{S_{i}(t+1)\right\} \mathscr{E}\left\{S_{j}(t+1)\right\} \mathscr{E}\left\{y_{i}(t)\right\} \mathscr{E}\left\{y_{j}(t)\right\} \\
= & \operatorname{Cov}\left\{S_{i}(t+1), S_{j}(t+1)\right\} \\
& \left.+\mathscr{E}\left\{S_{i}(t+1)\right\} \mathscr{E}\left\{S_{j}(t+1)\right\}\right] o_{i j}(t) \\
+ & \operatorname{Cov}\left\{S_{i}(t+1), S_{j}(t+1)\right\} \mathscr{E}\left\{y_{i}(t)\right\} \mathscr{E}\left\{y_{j}(t)\right\} .
\end{aligned}
$$

Using (3) and Lemma 3.1, this equation can be simplified to yield

$$
\begin{aligned}
& o_{i j}(t+1) \\
& =\left[\pi_{i j}(t+1)+\left(1-p_{i}(t+1)\right)\left(1-p_{j}(t+1)\right)\right] o_{i j}(t) \\
& +\pi_{i j}(t+1) \prod_{k=1}^{t}\left(1-p_{i}(k)\right) \prod_{k=1}^{t}\left(1-p_{j}(k)\right),
\end{aligned}
$$

which completes the proof of (13).

For $(14)$, since $y_{i}(0)=y_{j}(0)=1$, we have

$$
o_{i j}(0)=\operatorname{Cov}\left\{y_{i}(0), y_{j}(0)\right\}=0,
$$

as required.

Note that $o_{i j}(t)$ is independent of the allocation vector and can be computed immediately from equations (13) and (14).

Now, since the pension payment in period $t+1$ may depend on the pension payments in previous period$\mathrm{s}, x(t)$ and $\psi(t+1)$ are not necessarily independent. The covariance of $x(t)$ and $\psi(t+1)$ is given in the next result.

Lemma 3.3. For each $t=0, \ldots, T-1$, the covariance of $x(t)$ and $\psi(t+1)$ is given by

$$
\operatorname{Cov}\{x(t), \psi(t+1)\}=-\sum_{k=1}^{t} \sigma(k, t+1) .
$$

Proof. It is easy to prove by induction that

$$
\begin{array}{r}
x(t)=x(0)+\sum_{k=1}^{t} \sum_{i=1}^{N} \frac{K u_{i}}{\gamma_{i}} \alpha_{i}(k) y_{i}(k)-\sum_{k=1}^{t} \psi(k), \\
t=0, \ldots, T .
\end{array}
$$


Hence, since $y_{i}(k)$ and $\psi(t+1)$ are independent,

$$
\begin{array}{r}
\operatorname{Cov}\{x(t), \psi(t+1)\}=-\sum_{k=1}^{t} \sigma(k, t+1), \\
t=0, \ldots, T-1 .
\end{array}
$$

This completes the proof.

Let $z(t)=\mathscr{E}\{x(t)\}$ and $v(t)=\operatorname{Var}\{x(t)\}$. Furthermore, let $w_{i}(t), i=1, \ldots, N$, be new state variables defined as follows:

$$
w_{i}(t)=\operatorname{Cov}\left\{x(t), y_{i}(t+1)\right\}, \quad t=0, \ldots, T-1 .
$$

Taking the expectation of (4) and then applying (11) yields

$$
\begin{aligned}
& z(t+1)=\mathscr{E}\{x(t+1)\} \\
& =\mathscr{E}\left\{x(t)+\sum_{i=1}^{N} \frac{K u_{i}}{\gamma_{i}} \alpha_{i}(t+1) y_{i}(t+1)-\psi(t+1)\right\} \\
& =z(t)+\sum_{i=1}^{N} \frac{K u_{i}}{\gamma_{i}} \prod_{k=1}^{t+1}\left(1-p_{i}(k)\right) \alpha_{i}(t+1)-\mu(t+1), \\
& t=0, \ldots, T-1 .
\end{aligned}
$$

The initial condition for $z(t)$ is:

$$
z(0)=\mathscr{E}\left\{K\left[1-\sum_{i=1}^{N} u_{i}\right]\right\}=K\left[1-\sum_{i=1}^{N} u_{i}\right]
$$

Recall that $y_{i}(t+1)$ and $\psi(t+1)$ are independent. Thus, it follows from Lemmas 3.2 and 3.3 that the variance of $x(t)$ can be calculated as given below:

$$
\begin{aligned}
& v(t+1)=\operatorname{Var}\{x(t+1)\} \\
& =\operatorname{Var}\left\{x(t)+\sum_{i=1}^{N} \frac{K u_{i}}{\gamma_{i}} \alpha_{i}(t+1) y_{i}(t+1)-\psi(t+1)\right\} \\
& =v(t)+\sum_{i=1}^{N} \sum_{j=1}^{N} \frac{K^{2} u_{i} u_{j}}{\gamma_{i} \gamma_{j}} \alpha_{i}(t+1) \alpha_{j}(t+1) o_{i j}(t+1) \\
& \quad+2 \sum_{i=1}^{N} \frac{K u_{i}}{\gamma_{i}} \alpha_{i}(t+1) w_{i}(t) \\
& \quad+2 \sum_{k=1}^{t} \sigma(k, t+1)+\sigma(t+1, t+1), \\
& \quad t=0, \ldots, T-1
\end{aligned}
$$

with initial condition

$$
v(0)=\operatorname{Var}\left\{K\left[1-\sum_{i=1}^{N} u_{i}\right]\right\}=0 .
$$

Equations (15)-(18) define the dynamic equations for the state variables $z(t)$ and $v(t)$. We now derive the dynamic equations for $w_{i}(t)$.

Theorem 3.1. For each $i=1, \ldots, N$, the new state variable $w_{i}(t)$ satisfies the discrete-time system

$$
\begin{aligned}
w_{i}(t+1) & =\left(1-p_{i}(t+2)\right) w_{i}(t) \\
+ & \sum_{j=1}^{N} \frac{K u_{j}}{\gamma_{j}}\left(1-p_{i}(t+2)\right) \alpha_{j}(t+1) o_{i j}(t+1) \\
& t=0, \ldots, T-1
\end{aligned}
$$

with initial condition

$$
w_{i}(0)=0 .
$$

Proof. First, using (4),

$$
\begin{aligned}
& w_{i}(t+1)= \operatorname{Cov}\left\{x(t+1), y_{i}(t+2)\right\} \\
&=\operatorname{Cov}\left\{x(t)+\sum_{j=1}^{N} \frac{K u_{j}}{\gamma_{j}} \alpha_{j}(t+1) y_{j}(t+1)\right. \\
&\left.\quad-\psi(t+1), y_{i}(t+2)\right\} .
\end{aligned}
$$

Since $\psi(t+1)$ is independent of $y_{i}(t+2)$, and $x(t), y_{i}(t+1)$ and $y_{j}(t+1)$ are independent of $S_{i}(t+2)$, it follows from (1) that

$$
\begin{aligned}
w_{i}(t+1) & \operatorname{Cov}\left\{x(t), y_{i}(t+2)\right\} \\
& +\sum_{j=1}^{N} \frac{K u_{j}}{\gamma_{j}} \alpha_{j}(t+1) \operatorname{Cov}\left\{y_{j}(t+1), y_{i}(t+2)\right\} \\
= & \operatorname{Cov}\left\{x(t), S_{i}(t+2) y_{i}(t+1)\right\}+\sum_{j=1}^{N} \frac{K u_{j}}{\gamma_{j}} \\
& \cdot \alpha_{j}(t+1) \operatorname{Cov}\left\{y_{j}(t+1), S_{i}(t+2) y_{i}(t+1)\right\} \\
= & \mathscr{E}\left\{S_{i}(t+2)\right\} \operatorname{Cov}\left\{x(t), y_{i}(t+1)\right\}+\sum_{j=1}^{N} \frac{K u_{j}}{\gamma_{j}} \\
& \cdot \alpha_{j}(t+1) \mathscr{E}\left\{S_{i}(t+2)\right\} \operatorname{Cov}\left\{y_{j}(t+1), y_{i}(t+1)\right\} \\
= & \left(1-p_{i}(t+2)\right) w_{i}(t) \\
& +\sum_{j=1}^{N} \frac{K u_{j}}{\gamma_{j}}\left(1-p_{i}(t+2)\right) \alpha_{j}(t+1) o_{i j}(t+1),
\end{aligned}
$$

which proves (19). For (20), since $x(0)$ is a constant, we immediately have

$$
w_{i}(0)=\operatorname{Cov}\left\{x(0), y_{i}(1)\right\}=0,
$$


as required.

Now, consider the constraint

$$
z(t)-\left(\frac{q v(t)}{1-q}\right)^{\frac{1}{2}} \geq x_{\min }, t=0, \ldots, T,
$$

where $q$ and $x_{\min }$ are as defined in the chance constraint (9). Let

$$
\omega_{q}=\left(\frac{q}{1-q}\right)^{\frac{1}{2}} .
$$

Now, by the one-sided Chebyshev's inequality (Grimmett and Stirzaker (2001)), it follows that if (21) is satisfied, then

$$
\begin{aligned}
\operatorname{Pr}\left\{x(t)<x_{\min }\right\} & \leq \operatorname{Pr}\left\{x(t) \leq x_{\min }\right\} \\
& \leq \operatorname{Pr}\left\{x(t) \leq z(t)-\omega_{q} \sqrt{v(t)}\right\} \\
& \leq \frac{v(t)}{v(t)+\omega_{q}^{2} v(t)} .
\end{aligned}
$$

Hence,

$$
\begin{aligned}
\operatorname{Pr}\left\{x(t) \geq x_{\min }\right\} & =1-\operatorname{Pr}\left\{x(t)<x_{\min }\right\} \\
& \geq 1-\frac{v(t)}{v(t)+\omega_{q}^{2} v(t)} \\
& =\frac{\omega_{q}^{2}}{1+\omega_{q}^{2}}=q .
\end{aligned}
$$

Thus, constraint (21), which depends explicitly on the state variables $z(t)$ and $v(t)$, implies the chance constraint (9). We therefore use (21) as a conservative approximation of (9).

For the objective function, using (10) and (11) we have

$$
\begin{aligned}
g(u) & =\mathscr{E}\{x(T)\}+\sum_{i=1}^{N} \frac{K u_{i}}{\gamma_{i}} c_{i} \mathscr{E}\left\{y_{i}(T)\right\} \\
& =z(T)+\sum_{i=1}^{N} \frac{K u_{i}}{\gamma_{i}} c_{i} \prod_{k=1}^{T}\left(1-p_{i}(k)\right)
\end{aligned}
$$

On this basis, Problem $\mathrm{P}$ is approximated by the following discrete-time deterministic optimal control problem.

Problem $\hat{\mathbf{P}}$. Given the discrete-time system (15), (17) and (19) with initial conditions (16), (18) and (20), find an admissible allocation vector $u \in U$ such that the objective function

$$
g(u)=z(T)+\sum_{i=1}^{N} \frac{K u_{i}}{\gamma_{i}} c_{i} \prod_{k=1}^{T}\left(1-p_{i}(k)\right)
$$

is maximized subject to constraint (21).

Problem $\hat{\mathrm{P}}$ is a discrete-time deterministic optimal control problem. Note that Problem $\hat{P}$ only involves the first- and second-order moments of $x(t)$. This is because the deterministic constraint (21), derived using Chebyshev's inequality, only requires first- and second-order moments, even though the outgoing pension payments are of arbitrary distribution.

Using the notation $\omega_{q}$, constraint (21) can be rewritten as follows:

$$
z(t) \geq x_{\min }+\omega_{q} \sqrt{v(t)}, \quad t=0, \ldots, T .
$$

This means that the expected cash level must be $\omega_{q}$ standard deviations above the minimum threshold $x_{\min }$. For example, if $q=80 \%$, then we require the expected cash level to be $\omega_{q}=2$ standard deviations above the minimum; if $q=90 \%$, then we require $\omega_{q}=3 \mathrm{~s}-$ tandard deviations. This is more conservative than the normal distribution, where $95 \%$ of sample trajectories lie within two standard deviations and $99.7 \%$ of sample trajectories lie within three standard deviations. Recal1 , however, that the outgoing pension payments in our problem can be of any distribution, not just normal.

We now introduce a gradient-based optimization algorithm for solving Problem $\hat{P}$.

\section{Solving Problem $\hat{\mathbf{P}}$}

Problem $\hat{\mathrm{P}}$ includes a single all-time state constraint (21). This all-time state constraint is difficult to handle computationally because it is imposed at every point in the time horizon. Thus, in this section, we will use the constraint transcription method (Teo et al. (1991)) to approximate the all-time constraint (21) by a conventional constraint. We then develop a gradient-based optimization algorithm for solving the resulting approximation of Problem $\hat{P}$.

\subsection{Constraint transcription}

Let

$$
h(z(t), v(t))=z(t)-\left(\frac{q v(t)}{1-q}\right)^{\frac{1}{2}}-x_{\min } .
$$

Then constraint (21) is equivalent to the following equality constraint:

$$
\sum_{t=0}^{T} \min \{h(z(t), v(t)), 0\}=0 .
$$


Thus, the $T+1$ constraints given by (21) can be replaced by the single constraint (23). However, because of the $\min \{\cdot, 0\}$ function, the new constraint (23) is nonsmooth. This will cause problems for gradient-based optimization algorithms. Hence, we introduce the following smooth approximation for $\min \{\cdot, 0\}$ :

$$
\varphi_{\delta}(\theta)= \begin{cases}\theta, & \text { if } \theta<-\delta \\ -(\theta-\delta)^{2} / 4 \delta, & \text { if }-\delta \leq \theta \leq \delta \\ 0, & \text { if } \theta>\delta\end{cases}
$$

where $\delta$ is an adjustable parameter that controls the accuracy of the approximation (Lin et al. (2013)). Based on the smoothing function (24), we now define the following approximation for constraint (23):

$$
\frac{\delta}{4}+\sum_{t=0}^{T} \varphi_{\delta}(h(z(t), v(t))) \geq 0
$$

Our next result shows that (25) actually implies the alltime state constraint (21).

Theorem 4.1. Let $u \in U$ be an admissible allocation vector satisfying constraint (25). Then u also satisfies constraint (21).

Proof. Suppose that $u$ satisfies constraint (25), but not constraint (21). Then there exists a $t^{\prime} \in\{0, \ldots, T\}$ such that

$$
h\left(z\left(t^{\prime}\right), v\left(t^{\prime}\right)\right)<0 .
$$

Since $\varphi_{\delta}$ is strictly increasing on $(-\infty, 0]$, this implies that

$$
\varphi_{\delta}\left(h\left(z\left(t^{\prime}\right), v\left(t^{\prime}\right)\right)\right)<\varphi_{\delta}(0)=-\frac{\delta}{4} .
$$

Thus,

$$
\frac{\delta}{4}+\sum_{t=0}^{T} \varphi_{\delta}(h(z(t), v(t))) \leq \frac{\delta}{4}+\varphi_{\delta}\left(h\left(z\left(t^{\prime}\right), v\left(t^{\prime}\right)\right)\right)<0 .
$$

But this contradicts (25), and thus our initial assumption that (21) is violated must be false.

Based on the new constraint (25), the approximate problem for Problem $\hat{\mathrm{P}}$ can be stated formally as below.

Problem $\hat{\mathbf{P}}_{\delta}$. Given the discrete-time system (15), (17) and (19) with initial conditions (16), (18) and (20), find an admissible allocation vector $u \in U$ such that the $o b$ jective function (22) is maximized subject to constraint (25).

\subsection{Gradient formulae}

The key step to solving Problem $\hat{\mathrm{P}}_{\delta}$ using gradientbased optimization techniques is to determine the gra- dients of the objective function (22) and the constraint function (25). This is the aim of the present section.

We rewrite the dynamics in Problem $\hat{\mathrm{P}}_{\delta}$ in the following state-space form:

$$
\zeta(t+1)=f(t, \zeta(t), u), \quad t=0, \ldots, T-1,
$$

where $\zeta(t)=\left[z(t), v(t), w_{1}(t), \ldots, w_{N}(t)\right]^{\top} \in \mathbb{R}^{N+2}$ is the state vector, $u=\left[u_{1}, \ldots, u_{N}\right]^{\top} \in \mathbb{R}^{N}$ is the allocation vector, and

$$
\begin{gathered}
f(t, \zeta(t), u)= \\
\left(\begin{array}{c}
z(t)+\sum_{i=1}^{N} \frac{K u_{i}}{\gamma_{i}} \prod_{k=1}^{t+1}\left(1-p_{i}(k)\right) \alpha_{i}(t+1)-\mu(t+1) \\
v(t)+\sum_{i=1}^{N} \sum_{j=1}^{N} \frac{K^{2} u_{i} u_{j}}{\gamma_{i} \gamma_{j}} \alpha_{i}(t+1) \alpha_{j}(t+1) o_{i j}(t+1) \\
+2 \sum_{i=1}^{N} \frac{K u_{i}}{\gamma_{i}} \alpha_{i}(t+1) w_{i}(t) \\
+2 \sum_{k=1}^{t} \sigma(k, t+1)+\sigma(t+1, t+1) \\
\left(1-p_{1}(t+2)\right) w_{1}(t) \\
+\sum_{j=1}^{N} \frac{K u_{j}}{\gamma_{j}}\left(1-p_{1}(t+2)\right) \alpha_{j}(t+1) o_{1 j}(t+1) \\
\quad\left(1-p_{N}(t+2)\right) w_{N}(t) \\
+\sum_{j=1}^{N} \frac{K u_{j}}{\gamma_{j}}\left(1-p_{N}(t+2)\right) \alpha_{j}(t+1) o_{N j}(t+1)
\end{array}\right) .
\end{gathered}
$$

The derivative of $f$ with respect to $\zeta$ is the $(N+2) \times$ $(N+2)$ matrix given by

$$
\frac{\partial f(t, \zeta(t), u)}{\partial \zeta}=\left(\begin{array}{ll}
I & \Gamma \\
0 & \Lambda
\end{array}\right)
$$

where $I$ is the $2 \times 2$ identity matrix, 0 is the $N \times 2$ zero matrix, and

$$
\begin{aligned}
\Gamma & =\left(\begin{array}{ccc}
0 & \ldots & 0 \\
\frac{2 K u_{1}}{\gamma_{1}} \alpha_{1}(t+1) & \ldots & \frac{2 K u_{N}}{\gamma_{N}} \alpha_{N}(t+1)
\end{array}\right), \\
\Lambda & =\operatorname{diag}\left(1-p_{1}(t+2), \ldots, 1-p_{N}(t+2)\right) .
\end{aligned}
$$

Moreover, the derivative of $f$ with respect to $u$ is the $(N+2) \times N$ matrix given by

$$
\frac{\partial f(t, \zeta(t), u)}{\partial u}=\left(\begin{array}{l}
\Omega_{1} \\
\Omega_{2} \\
\Omega_{3}
\end{array}\right)
$$


where

$$
\begin{aligned}
& \Omega_{1}=\frac{\partial f_{1}(t, \zeta(t), u)}{\partial u}, \\
& \Omega_{2}=\frac{\partial f_{2}(t, \zeta(t), u)}{\partial u}, \\
& \Omega_{3}=\left[\frac{\partial f_{i}(t, \zeta(t), u)}{\partial u}\right]_{i=3, \ldots, N+2} .
\end{aligned}
$$

More specifically,

$$
\begin{aligned}
\Omega_{1}^{\top}= & \left(\begin{array}{c}
\frac{K}{\gamma_{1}} \prod_{k=1}^{t+1}\left(1-p_{1}(k)\right) \alpha_{1}(t+1) \\
\vdots \\
\frac{K}{\gamma_{N}} \prod_{k=1}^{t+1}\left(1-p_{N}(k)\right) \alpha_{N}(t+1)
\end{array}\right), \\
\Omega_{2}^{\top}= & \left(\begin{array}{c}
\sum_{i=1}^{N} \frac{2 K^{2} u_{i}}{\gamma_{i} \gamma_{1}} \alpha_{i}(t+1) \alpha_{1}(t+1) o_{i 1}(t+1) \\
+\frac{2 K}{\gamma_{1}} \alpha_{1}(t+1) w_{1}(t) \\
\vdots \\
\sum_{i=1}^{N} \frac{2 K^{2} u_{i}}{\gamma_{i} \gamma_{N}} \alpha_{i}(t+1) \alpha_{N}(t+1) o_{i N}(t+1) \\
+\frac{2 K}{\gamma_{N}} \alpha_{N}(t+1) w_{N}(t)
\end{array}\right),
\end{aligned}
$$

and $\Omega_{3}$ is a $N \times N$ matrix whose $(i, j)^{\text {th }}$ element is given by

$$
\Omega_{3}(i, j)=\frac{K}{\gamma_{j}}\left(1-p_{i}(t+2)\right) \alpha_{j}(t+1) o_{i j}(t+1) .
$$

The initial condition for (26) is

$$
\zeta(0)=\left(\begin{array}{c}
K\left[1-\sum_{i=1}^{N} u_{i}\right] \\
0 \\
0 \\
\vdots \\
0
\end{array}\right)
$$

Note that the objective function (22) and the constraint function (25) can be expressed in the following unified form:

$$
J(u)=\Phi(\zeta(T), u)+\sum_{t=0}^{T-1} \mathscr{L}(\zeta(t)),
$$

where $\Phi: \mathbb{R}^{N+2} \times \mathbb{R}^{N} \rightarrow \mathbb{R}$ is a function of the state and allocation vectors, and $\mathscr{L}: \mathbb{R}^{N+2} \rightarrow \mathbb{R}$ is a function of the state vector. For the objective function (22), the functions $\Phi$ and $\mathscr{L}$ in (30) are

$$
\begin{aligned}
\Phi(\zeta(T), u) & =z(T)+\sum_{i=1}^{N} \frac{K u_{i}}{\gamma_{i}} c_{i} \prod_{k=1}^{T}\left(1-p_{i}(k)\right), \\
\mathscr{L}(\zeta(t)) & =0 .
\end{aligned}
$$

For the constraint function (25), the functions $\Phi$ and $\mathscr{L}$ in (30) are

$$
\begin{aligned}
\Phi(\zeta(T), u) & =\frac{\delta}{4}+\varphi_{\delta}(h(z(T), v(T))) \\
\mathscr{L}(\zeta(t)) & =\varphi_{\delta}(h(z(t), v(t)))
\end{aligned}
$$

We now derive formulae for computing the gradient of (30) with respect to $u$. We will then use these formulae to determine the gradients of the objective and constraint functions in Problem $\hat{\mathrm{P}}_{\delta}$.

Consider the following perturbed allocation vector:

$$
u^{\varepsilon}=u+\varepsilon \hat{u},
$$

where $\hat{u}$ is an arbitrary but fixed perturbation and $\varepsilon$ is a real number of sufficiently small magnitude. Let $\zeta(\cdot)$ denote the solution of (26) and (29) corresponding to the unperturbed allocation vector $u$, and let $\zeta^{\varepsilon}(\cdot)$ denote the solution corresponding to the perturbed allocation vector $u^{\varepsilon}$.

For each $t=0, \ldots, T-1$, the variation of the state is:

$$
\begin{aligned}
\Delta \zeta(t+1) & =\left.\frac{d \zeta^{\varepsilon}(t+1)}{d \varepsilon}\right|_{\varepsilon=0} \\
& =\left.\frac{d}{d \varepsilon}\left\{f\left(t, \zeta^{\varepsilon}(t), u+\varepsilon \hat{u}\right)\right\}\right|_{\varepsilon=0} \\
& =\frac{\partial f(t, \zeta(t), u)}{\partial \zeta} \Delta \zeta(t)+\frac{\partial f(t, \zeta(t), u)}{\partial u} \hat{u}
\end{aligned}
$$

where $\partial f / \partial \zeta$ and $\partial f / \partial u$ are defined in (27) and (28), respectively. At $t=0$, we have

$$
\Delta \zeta(0)=\left.\frac{d \zeta^{\varepsilon}(0)}{d \varepsilon}\right|_{\varepsilon=0}=\left(\begin{array}{c}
-K \sum_{i=1}^{N} \hat{u}_{i} \\
0 \\
0 \\
\vdots \\
0
\end{array}\right)=\kappa \hat{u}
$$


where $\kappa$ is a $(N+2) \times N$ matrix defined by

$$
\kappa=\left(\begin{array}{ccc}
-K & \cdots & -K \\
0 & \cdots & 0 \\
0 & \cdots & 0 \\
\vdots & \ddots & \vdots \\
0 & \cdots & 0
\end{array}\right)
$$

Thus,

$$
\begin{aligned}
\frac{\partial J(u)}{\partial u} \hat{u}= & \left.\frac{d J\left(u^{\varepsilon}\right)}{d \varepsilon}\right|_{\varepsilon=0} \\
= & \frac{\partial \Phi(\zeta(T), u)}{\partial \zeta} \Delta \zeta(T)+\frac{\partial \Phi(\zeta(T), u)}{\partial u} \hat{u} \\
& +\sum_{t=0}^{T-1} \frac{\partial \mathscr{L}(\zeta(t))}{\partial \zeta} \Delta \zeta(t)
\end{aligned}
$$

Now, define the Hamiltonian function as follows:

$$
\begin{gathered}
H(t, \zeta(t), u, \lambda(t+1))= \\
\qquad=0, \ldots, T-1,
\end{gathered}
$$

where $\lambda(t) \in \mathbb{R}^{N+2}$ is the costate sequence defined by

$$
\begin{array}{r}
(\lambda(t))^{\top}=\frac{\partial H(t, \zeta(t), u, \lambda(t+1))}{\partial \zeta}, \\
t=T-1, T-2, \ldots, 0,
\end{array}
$$

with final time condition

$$
(\lambda(T))^{\top}=\frac{\partial \Phi(\zeta(T), u)}{\partial \zeta}
$$

Now, using (38), we can rewrite (37) as follows:

$$
\begin{aligned}
\frac{\partial J(u)}{\partial u} \hat{u}= & \frac{\partial \Phi(\zeta(T), u)}{\partial \zeta} \Delta \zeta(T)+\frac{\partial \Phi(\zeta(T), u)}{\partial u} \hat{u} \\
& +\sum_{t=0}^{T-1}\left\{\frac{\partial H(t, \zeta(t), u, \lambda(t+1))}{\partial \zeta} \Delta \zeta(t)\right. \\
& \left.-(\lambda(t+1))^{\top} \frac{\partial f(t, \zeta(t), u)}{\partial \zeta} \Delta \zeta(t)\right\} .
\end{aligned}
$$

Hence, using (39) and (40), we obtain

$$
\begin{aligned}
\frac{\partial J(u)}{\partial u} \hat{u}= & (\lambda(T))^{\top} \Delta \zeta(T)+\frac{\partial \Phi(\zeta(T), u)}{\partial u} \hat{u} \\
& +\sum_{t=1}^{T-1}\left\{(\lambda(t))^{\top} \Delta \zeta(t)\right. \\
& \left.-(\lambda(t))^{\top} \frac{\partial f(t-1, \zeta(t-1), u)}{\partial \zeta} \Delta \zeta(t-1)\right\} \\
& +(\lambda(0))^{\top} \Delta \zeta(0) \\
& -(\lambda(T))^{\top} \frac{\partial f(T-1, \zeta(T-1), u)}{\partial \zeta} \Delta \zeta(T-1) .
\end{aligned}
$$

Thus, applying (35) and (36),

$$
\begin{aligned}
\frac{\partial J(u)}{\partial u} \hat{u}= & (\lambda(T))^{\top}\left\{\frac{\partial f(T-1, \zeta(T-1), u)}{\partial \zeta} \Delta \zeta(T-1)\right. \\
& \left.+\frac{\partial f(T-1, \zeta(T-1), u)}{\partial u} \hat{u}\right\} \\
& +\sum_{t=1}^{T-1}\left\{( \lambda ( t ) ) ^ { \top } \left[\frac{\partial f(t-1, \zeta(t-1), u)}{\partial \zeta} \Delta \zeta(t-1)\right.\right. \\
& \left.+\frac{\partial f(t-1, \zeta(t-1), u)}{\partial u} \hat{u}\right] \\
& \left.-(\lambda(t))^{\top} \frac{\partial f(t-1, \zeta(t-1), u)}{\partial \zeta} \Delta \zeta(t-1)\right\} \\
& +\frac{\partial \Phi(\zeta(T), u)}{\partial u} \hat{u}+(\lambda(0))^{\top} \Delta \zeta(0) \\
& -(\lambda(T))^{\top} \frac{\partial f(T-1, \zeta(T-1), u)}{\partial \zeta} \Delta \zeta(T-1) \\
= & \sum_{t=0}^{T-1}(\lambda(t+1))^{\top} \frac{\partial f(t, \zeta(t), u)}{\partial u} \hat{u} \\
& +\frac{\partial \Phi(\zeta(T), u)}{\partial u} \hat{u}+(\lambda(0))^{\top} \Delta \zeta(0) \\
& +\sum_{t=0}^{T-1} \frac{\partial H(t, \zeta(t), u, \lambda(t+1))}{\partial u} \hat{u} \\
& \frac{\partial \Phi(\zeta(T), u)}{\partial u} \hat{u}+(\lambda(0))^{\top} \kappa \hat{u} .
\end{aligned}
$$

Recall that $\hat{u}$ was selected arbitrarily. By choosing $\hat{u}$ to be the standard unit basis vectors, we obtain the following result.

Theorem 4.2. The gradient of $J(u)$ is given by

$$
\begin{aligned}
\frac{\partial J(u)}{\partial u}= & \sum_{t=0}^{T-1} \frac{\partial H(t, \zeta(t), u, \lambda(t+1))}{\partial u} \\
& +\frac{\partial \Phi(\zeta(T), u)}{\partial u}+(\lambda(0))^{\top} \kappa .
\end{aligned}
$$


Now, using the formula in Theorem 4.2, we can express the gradient of the cost function (22) as follows:

$$
\begin{aligned}
& \frac{\partial g(u)}{\partial u}=\sum_{t=0}^{T-1}(\lambda(t+1))^{\top} \frac{\partial f(t, \zeta(t), u)}{\partial u} \\
& \quad+\left[\frac{K c_{1}}{\gamma_{1}} \prod_{k=1}^{T}\left(1-p_{1}(k)\right) \cdots \frac{K c_{N}}{\gamma_{N}} \prod_{k=1}^{T}\left(1-p_{N}(k)\right)\right] \\
& \quad+(\lambda(0))^{\top} \kappa
\end{aligned}
$$

where $\partial f / \partial u$ is given by (28).

Similarly, the gradient of the constraint function (25) is given by

$$
\frac{\partial J(u)}{\partial u}=\sum_{t=0}^{T-1}(\lambda(t+1))^{\top} \frac{\partial f(t, \zeta(t), u)}{\partial u}+(\lambda(0))^{\top} \kappa .
$$

The gradient formulae in (41) and (42) can be readily incorporated into a standard gradient-based optimization algorithm to solve Problem $\hat{\mathrm{P}}_{\delta}$. Note that the costate sequences for (41) and (42) are different. For (41), the costate system (39) and (40) has $\Phi$ and $\mathscr{L}$ defined by (31) and (32); for (42), the costate system (39) and (40) has $\Phi$ and $\mathscr{L}$ defined by (33) and (34).

\section{Numerical simulations}

In this section, we consider a randomly-generated test problem and give the corresponding optimal portfolios for $u_{i}^{\max }=5 \%$ and $u_{i}^{\max }=10 \%$.

Suppose that our fictional pension fund has $K=$ $1,000,000$ dollars of initial capital to invest. Suppose also that the portfolio is constructed from $N=10$ different bond types. The aim is to determine an optimal bond allocation such that the terminal wealth of the portfolio is maximized at the end of the planning horizon, which is assumed to be $T=12$ months. The parameter$\mathrm{s}$ for the all-time chance constraint are $x_{\min }=200,000$ and $q=0.8$.

The pension fund makes payments at the end of each monthly period. These payments $\psi(t), t=1, \ldots, 12$, are normally-distributed random variables with means and covariances given in Tables 1 and 2, respectively. The means in Table 1 were selected randomly from the interval $[30000,60000]$. For the covariance matrix in Table 2 , the diagonal elements were first selected randomly from the interval $\left[0,10^{8}\right]$. Then, for each off-diagonal element $\left(t, t^{\prime}\right)$, the corresponding correlation coefficient Corr $\left(\psi(t), \psi\left(t^{\prime}\right)\right)$ was randomly selected from the in-
Table 1: Mean of the monthly pension payment

\begin{tabular}{ccccc}
\hline$t$ & 1 & 2 & 3 & 4 \\
\hline$\mu(t)$ & 46640 & 49024 & 54891 & 35668 \\
\hline$t$ & 5 & 6 & 7 & 8 \\
\hline$\mu(t)$ & 54890 & 58276 & 39005 & 46566 \\
\hline$t$ & 9 & 10 & 11 & 12 \\
\hline$\mu(t)$ & 36327 & 50734 & 43152 & 58798 \\
\hline
\end{tabular}

Table 3: Price, coupon, par value, and default probability of each bond type

\begin{tabular}{cccccc}
\hline Bond $i$ & $\gamma_{i}$ & $\alpha_{i}$ & $c_{i}$ & $p_{i}(t)$ & $c_{i}^{\prime}$ \\
\hline TB120 & 110.830 & 0.500 & 110.885 & $0.450 \%$ & 109.114 \\
TB135 & 104.981 & 0.354 & 105.088 & $0.275 \%$ & 104.038 \\
TB132 & 110.406 & 0.458 & 110.518 & $0.400 \%$ & 108.786 \\
TB122 & 110.541 & 0.438 & 110.783 & $0.375 \%$ & 108.747 \\
TB126 & 106.528 & 0.375 & 106.859 & $0.300 \%$ & 105.003 \\
TB124 & 114.819 & 0.479 & 115.264 & $0.425 \%$ & 112.443 \\
TB128 & 115.596 & 0.479 & 116.143 & $0.425 \%$ & 112.772 \\
TB133 & 114.208 & 0.458 & 114.786 & $0.400 \%$ & 111.258 \\
TB137 & 90.342 & 0.229 & 90.882 & $0.125 \%$ & 88.327 \\
TB136 & 106.852 & 0.396 & 107.700 & $0.325 \%$ & 103.754 \\
\hline
\end{tabular}

terval $[0,0.1]$ with the covariance computed by

$$
\begin{aligned}
\operatorname{Cov}\left(\psi(t), \psi\left(t^{\prime}\right)\right)= & \operatorname{Corr}\left(\psi(t), \psi\left(t^{\prime}\right)\right) \\
& \times \operatorname{Var}(\psi(t)) \operatorname{Var}\left(\psi\left(t^{\prime}\right)\right) .
\end{aligned}
$$

Each bond type $i$ is defined by its price $\gamma_{i}$, monthly coupon value $\alpha_{i}$, and par value $c_{i}$ (selling price). We consider 10 real bonds issued by the Australian government. The data for each bond, obtained from the Reserve Bank of Australia, is given in Table 3. Note that $c_{i}$ in Table 3 is the redemption value of bond type $i$ at time $t=0$ and $c_{i}^{\prime}$ is the updated redemption value after $t=6$ months. Both par values were obtained from historical data in the period 2012-2013. We assume that the default risk is time-invariant for each bond. Hence, the default probabilities $p_{i}(t)$ are constant with respect to $t$. These probabilities are linear functions of the rate of return. The time-invariant covariance matrix $\pi_{i j}(t)$ given in Table 4 was generated by choosing the diagonal entries to be $\operatorname{Var}\left(S_{i}(t)\right)=p_{i}(t)\left(1-p_{i}(t)\right)$ with the offdiagonal entries generated randomly in the same way as Table 2. Note that these bonds would be more strongly correlated in reality since they are from the same issuer. However, the randomly-generated data used here is sufficient for the purposes of our numerical simulations. 
Table 2: Covariance matrix for the monthly pension payments $\psi(t), t=1, \ldots, 12$

\begin{tabular}{|c|c|c|c|c|c|c|c|c|c|c|c|c|}
\hline & $\psi(1)$ & $\psi(2)$ & $\psi(3)$ & $\psi(4)$ & $\psi(5)$ & $\psi(6)$ & $\psi(7)$ & $\psi(8)$ & $\psi(9)$ & $\psi(10)$ & $\psi(11)$ & $\psi(12)$ \\
\hline$\psi(1)$ & 28397556 & 1556668 & 15488442 & 7010829 & 2546108 & 77702 & 1234685 & 275104 & 171189 & 7041 & 20372 & 3526 \\
\hline$\psi(2)$ & & 90990339 & 3944187 & 60307276 & 10162245 & 2333316 & 7752 & 1603968 & 1817158 & 61762 & 4248821 & 1231959 \\
\hline$\psi(3)$ & & & 24955656 & 4344821 & 665348 & 13992 & 452203 & 31848 & 11907 & 2278 & 35 & 34 \\
\hline$\psi(4)$ & & & & 95438219 & 10224014 & 63785 & 950575 & 357385 & 9318912 & 1535112 & 419701 & 712424 \\
\hline$\psi(5)$ & & & & & 70127631 & 240042 & 577217 & 1034195 & 3592402 & 4971 & 1035472 & 385638 \\
\hline$\psi(6)$ & & & & & & 3496194 & 91912 & 132622 & 18783 & 47748 & 469304 & 5369 \\
\hline$\psi(7)$ & & & & & & & 3322735 & 4231 & 3813 & 140 & 145 & 21 \\
\hline$\psi(8)$ & & & & & & & & 15445233 & 11587672 & 3802 & 193525 & 108567 \\
\hline$\psi(9)$ & & & & & & & & & 95215466 & 327 & 43074462 & 1805579 \\
\hline$\psi(10)$ & & & & & & & & & & 1663471 & 110 & 145 \\
\hline$\psi(11)$ & & & & & & & & & & & 82714753 & 687550 \\
\hline$\psi(12)$ & & & & & & & & & & & & 5022122 \\
\hline
\end{tabular}

Table 4: Covariance matrix for $S_{i}(t), i=1, \ldots, N$

\begin{tabular}{lcccccccccc}
\hline Bond $i$ & TB120 & TB135 & TB132 & TB122 & TB126 & TB124 & TB128 & TB133 & TB137 & TB136 \\
\hline TB120 & $0.44797 \%$ & $0.00127 \%$ & $0.02436 \%$ & $0.03327 \%$ & $0.02365 \%$ & $0.03422 \%$ & $0.03732 \%$ & $0.01765 \%$ & $0.00004 \%$ & $0.02464 \%$ \\
TB135 & & $0.27424 \%$ & $0.01013 \%$ & $0.02073 \%$ & $0.01493 \%$ & $0.02038 \%$ & $0.01754 \%$ & $0.00129 \%$ & $0.00395 \%$ & $0.02791 \%$ \\
TB132 & & & $0.39840 \%$ & $0.02019 \%$ & $0.01951 \%$ & $0.03760 \%$ & $0.02590 \%$ & $0.02862 \%$ & $0.00638 \%$ & $0.00493 \%$ \\
TB122 & & & & $0.37359 \%$ & $0.00672 \%$ & $0.03642 \%$ & $0.02265 \%$ & $0.01565 \%$ & $0.01609 \%$ & $0.00054 \%$ \\
TB126 & & & & & $0.29910 \%$ & $0.02069 \%$ & $0.02090 \%$ & $0.00036 \%$ & $0.01057 \%$ & $0.02820 \%$ \\
TB124 & & & & & $0.42319 \%$ & $0.02056 \%$ & $0.03403 \%$ & $0.01391 \%$ & $0.01839 \%$ \\
TB128 & & & & & & $0.42319 \%$ & $0.01750 \%$ & $0.00885 \%$ & $0.02767 \%$ \\
TB133 & & & & & & & $0.39840 \%$ & $0.01656 \%$ & $0.01275 \%$ \\
TB137 & & & & & & & & $0.12484 \%$ & $0.01667 \%$ \\
TB136 & & & & & & & & & & $0.32394 \%$ \\
\hline
\end{tabular}

We obtained the deterministic problem (i.e., Problem $\hat{\mathrm{P}}_{\delta}$ ) by applying the approximation techniques described in Sections 3 and 4. We then used the MATLAB gradient-based optimization function fmincon to solve the deterministic problem for $u_{i}^{\max }=5 \%$ and $u_{i}^{\max }=$ $10 \%$.

For $u_{i}^{\max }=5 \%$, the optimal value of the objective function is $4.2836 \times 10^{5}$ under redemption values $c_{i}$, and $4.2414 \times 10^{5}$ under redemption values $c_{i}^{\prime}$. For $u_{i}^{\max }=10 \%$, the objective function is $4.2906 \times 10^{5}$ under $c_{i}$, and $4.2375 \times 10^{5}$ under $c_{i}^{\prime}$. The optimal bond allocations $\left(u_{i}^{*}, i=1, \ldots, 10\right)$ are given in Table 5 . Note that the optimal portfolio for $u_{i}^{\max }=5 \%$ includes only four bond types and the optimal portfolio for $u_{i}^{\max }=$ $10 \%$ includes only two bond types. The optimization procedure has successfully identified the most attractive bonds and included them in the portfolio. For more diversification, a lower bound could be easily imposed on each bond's allocation, but this was not considered in the present work.

In comparison, for the naive $1 / N$ investment strategy (Demiguel et al. (2009a,b)), the resulting expected cash balance in the pension fund is $4.2347 \times 10^{5}$ with $u_{i}^{\max }=5 \%$, and $4.2348 \times 10^{5}$ with $u_{i}^{\max }=10 \%$. This is slightly less than the optimized portfolio. More importantly, the $1 / N$ strategy is actually infeasible because the expected cash balance falls below the minimum threshold $x_{\min }$ during the time horizon.

The results in the previous paragraph are for the $1 / N$ portfolio in which the entire initial capital $K$ is divided equally among the $N$ bonds. Other $1 / N$ portfolios can be constructed by investing less than $K$. In Table 6 , we give the expected fund value under $c_{i}^{\prime}$ for the $1 / N$ portfolios constructed by investing different initial amounts. Only one of the $1 / N$ portfolios (the $1 / N$ portfolio with $\$ 100,000$ invested) is feasible. This result is consistent with our optimized bond portfolios, which invest less than $\$ 200,000$ of the initial capital. Note that our optimization procedure determines both the optimal amount of initial investment and the optimal bond allocation such that the resulting combination of bonds maximizes the pension fund value while mitigating default risk. As shown in Table 6, where it is reported that most of the $1 / N$ portfolios are infeasible, designing the best $1 / N$ portfolio requires an optimization procedure to determine the best initial investment amount. Naively investing the entire capital may produce an infeasible result.

The optimal trajectories of the state variables $z(t)$ and $v(t)$ are illustrated in Figures 5.1 and 5.2, respec- 


\begin{tabular}{ccc}
\hline & \multicolumn{2}{c}{ Optimal Allocation } \\
\cline { 2 - 3 } Bond & $u_{i}^{\max }=5 \%$ & $u_{i}^{\max }=10 \%$ \\
\hline TB120 & $0.0000 \%$ & $0.0000 \%$ \\
TB135 & $2.1531 \%$ & $0.0000 \%$ \\
TB132 & $0.0000 \%$ & $0.0000 \%$ \\
TB122 & $0.0000 \%$ & $0.0000 \%$ \\
TB126 & $5.0000 \%$ & $0.0000 \%$ \\
TB124 & $0.0000 \%$ & $0.0000 \%$ \\
TB128 & $0.0000 \%$ & $0.0000 \%$ \\
TB133 & $0.0000 \%$ & $0.0000 \%$ \\
TB137 & $5.0000 \%$ & $10.0000 \%$ \\
TB136 & $5.0000 \%$ & $7.1028 \%$ \\
\hline
\end{tabular}

Table 5: Optimal bond allocations for the example in Section 5

tively. The optimal trajectories of the standard deviation $\sqrt{v(t)}$ are shown in Figure 5.3 for the two scenarios with different allocation limits. Figures 5.4 and 5.5 show simulated trajectories of $x(t)$ under 10,000 random scenarios for the optimized portfolios with $u_{i}^{\max }=$ $5 \%$ and $10 \%$, respectively. These simulations were performed using the internal MATLAB function $m v n$ rnd for sampling the normally-distributed pension payments $\psi(t)$ and the method in Park et al. (1996) for sampling the Bernoulli random variables $S_{i}(t)$.

In Figure 5.1, it is observed that the mean of the pension fund value decreases over time, as the pension payments in each period exceed the coupons received. At the end of the last period, there is a large increase in the fund value in each portfolio, which is due to the capital repayment for the outstanding bonds.

In Figure 5.2, the variance of the pension fund increases over time. The standard deviation of the pension fund, which is shown in Figure 5.3, follows a similar pattern to the variance.

Figures 5.4 and 5.5 show simulated trajectories of $x(t)$. The pattern is similar to the mean portfolio value shown in Figure 5.1. The variation for the first 11 months is due to the uncertainty in the pension payments. There is a possibility that some bonds default and this is reflected in the sample paths, which show a decline in pension fund value at the end of the investment horizon as the repayment of capital is lost.

\section{Conclusion}

In this paper, we have considered a portfolio optimization problem for a pension fund consisting of both government and corporate bonds, with the possibility of
Figure 5.1: Optimal mean profile $z(t)$

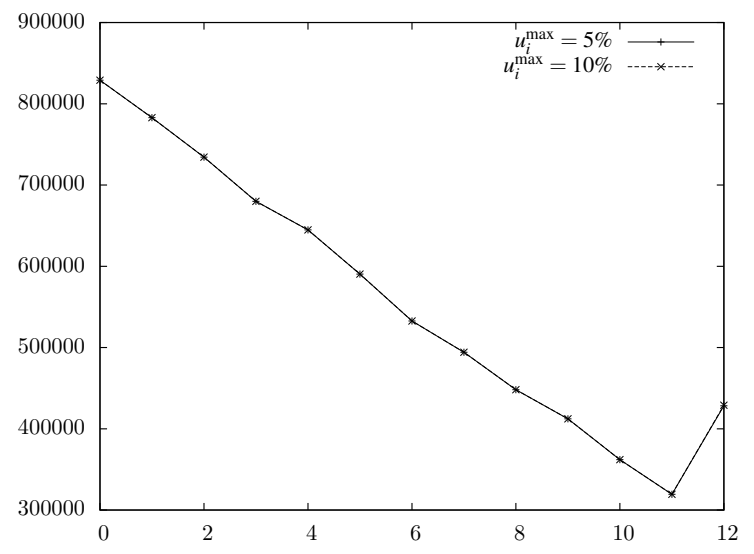

Figure 5.2: Optimal variance profile $v(t)$

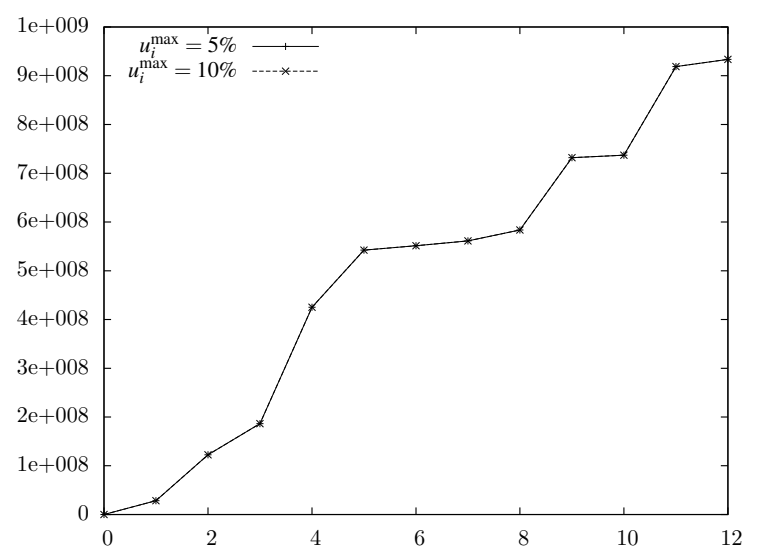

Figure 5.3: Optimal standard deviation profile $\sqrt{v(t)}$

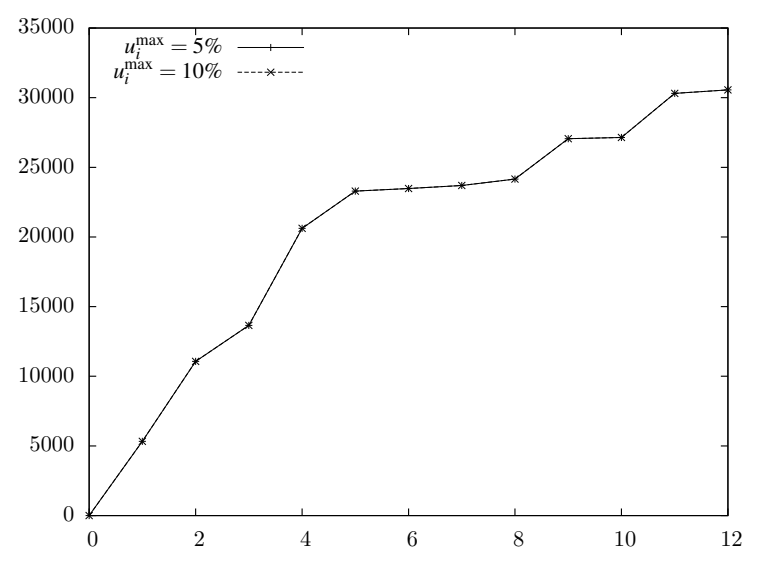


Figure 5.4: 10,000 sample paths for the fund cash level $\left(u_{i}^{\max }=5 \%\right)$

Figure 5.5: 10,000 sample paths for the fund cash level $\left(u_{i}^{\max }=10 \%\right)$

Table 6: Performance of the $1 / N$ portfolio for the example in Section 5

\begin{tabular}{ccc}
\hline Amount Invested & Expected Final Value & Feasible? \\
\hline$\$ 100,000$ & $\$ 424,537$ & Yes \\
$\$ 200,000$ & $\$ 423,047$ & No \\
$\$ 300,000$ & $\$ 421,556$ & No \\
$\$ 400,000$ & $\$ 420,066$ & No \\
$\$ 500,000$ & $\$ 418,575$ & No \\
$\$ 600,000$ & $\$ 417,085$ & No \\
$\$ 700,000$ & $\$ 415,594$ & No \\
$\$ 800,000$ & $\$ 414,104$ & No \\
$\$ 900,000$ & $\$ 412,613$ & No \\
$\$ 1,000,000$ & $\$ 411,123$ & No \\
\hline
\end{tabular}

bond defaults. The objective is to find the optimal investment strategy that maximizes the expected fund value at the end of the planning horizon. The problem is formulated as a discrete-time stochastic optimal control problem. The numerical simulations in Section 5 show that our proposed model gives solutions that, unlike the naive $1 / N$ investment strategy, are guaranteed to satisfy the robust all-time chance constraint. Note that we have assumed the portfolio composition remains static throughout the time horizon, with all investment decisions made at time $t=0$. Future research will involve problems in which the portfolio can be updated dynamically, taking into account transaction costs.

\section{Acknowledgement}

This work was partially supported by the National Science Foundation of China through International Young Scientists Research Fund 11350110208.

Çanakoğlu, E., \& Öekici, S. (2012). HARA frontiers of optimal portfolios in stochastic markets. European Journal of Operational Research, 221, 129-137.

Chen, Z. P., \& Song, Z. X. (2012). Dynamic portfolio optimization under multi-factor model in stochastic markets. OR Spectrum, 34, 885-919.

Çlikyurt, U., \& Öekici, S. (2007). Multiperiod portfolio optimization models in stochastic markets using the mean-variance approach. European Journal of Operational Research, 179, 186-202.

Demiguel, V., Garlappi, L., Nogales, F. J., \& Uppal, R. (2009a). A generalized approach to portfolio optimization: Improving performance by constraining portfolio norms. Management Science, 55, $798 \mathrm{C} 812$.

Demiguel, V., Garlappi, L., \& Uppal, R. (2009b). Optimal versus naive diversification: How inefficient is the $1 / N$ portfolio strategy? The Review of Financial Studies, 22, 1915-1953.

Duffie, D., \& Singleton, K. J. (2003). Credit Risk: Pricing, Measurement, and Management. Princeton: Princeton University Press.

Fama, E. F. (1970). Multiperiod consumption-investment decisions. The American Economic Review, 66, 723-724.

Grimmett, G. R., \& Stirzaker, D. R. (2001). Probability and Random Processes. Oxford: Oxford University Press.

Jarrow, R. A., \& Turnbull, S. M. (1995). Pricing derivatives on financial securities subject to credit risk. The Journal of Finance, 50, 53-85.

Lando, D. (2004). Credit Risk Modeling: Theory and Applications. Princeton: Princeton University Press.

Lin, Q., Loxton, R., \& Teo, K. L. (2013). Optimal control of nonlinear switched systems: Computational methods and applications. Journal of the Operations Research Society of China, 1, 275-311.

Markowitz, H. M. (1959). Portfolio Selection: Efficient Diversification of Investment. New York: John Wiley \& Sons.

Merton, R. C. (1969). Lifetime portfolio selection under uncertainty: The continuous-time case. Finance and Stochastics, 2, 173-198.

Mossin, J. (1968). Optimal multiperiod portfolio policies. Journal of Business, 41, 215-229.

Park, C. G., Park, T., \& Shin, D. W. (1996). A simple method for generating correlated binary variates. The American Statistician, 50, 306-310. 
Pliska, S. R. (1997). Introduction to Mathematical Finance. Malden: Basil Blackwell.

Schweizer, M. (1995). Variance-optimal hedging in discrete time Mathematics of Operations Research, 20, 1-32.

Smith, K. V. (1967). A transition model for portfolio revision. Journal of Finance, 22, 425-439.

Teo, K. L., Goh, C. J., \& Wong, K. H. (1991). A Unified Computational Approach to Optimal Control Problems. Essex: Longman Scientific and Technical.

Van Weert, K., Dhaene, J., \& Goovaerts, M. (2010). Optimal portfolio selection for general provisioning and terminal wealth problems. Insurance Mathematics and Economics, 47, 90-97.

Zhang, X. L., Zhang, K. C., \& Yu, X. J. (2009). Optimal proportiona reinsurance and investment with transaction costs, I: Maximizing the terminal wealth. Insurance Mathematics and Economics, 44, 473-478.

Zhu, S. S., Li, D., \& Wang, S. Y. (2004). Risk control over bankruptcy in dynamic portfolio selection: A generalized mean-variance formulation. IEEE Transactions on Automatic Control, 49, 447-457. 